\title{
Pulmonary Complications and Critical Care in Cancer Patients
}

\author{
Dr. B. A. Darmi, MD
}

\section{Introduction}

Cancer refers to the condition where abnormal proliferation of cells occurs overriding the natural check of cell death.[1] As a normal physiological process, all cells of our body are programmed to die called as apoptosis.[2] In cancer, the balance between cell proliferation and cell death is disrupted, resulting in uncontrolled division of cells.[3] Hence, apoptosis is an anti-cancer defense of body. In other words, carcinogenesis involves sequential genetic change that transforms normal cells to malignant cells. The cancer cells interfere with normal physiological functions and kill by invading various body organs vital for living. Cancer is a second leading cause of death worldwide, estimated to kill 9.6 million people in 2018.[4] Approximately, every sixth death is caused by cancer.[4] Globally, most common cancers are lung, breast, colorectal, prostate, skin and stomach cancers.

From the diagnosis to the treatment completion, cancer is a heartrending experience for the patients as well as for their families. Cancer poses a great psychosocial impact including clinical stress, severe anxiety and depression on the patient, his/her spouse, offspring and other family members. The patient faces physical and mental health challenges during the treatment as well as throughout rest of the life, affecting quality of life.[5] Moreover, psychosocial stress adds to the disability of cancer patients. Therefore, the family and the treating clinicians should be aware of psychosocial problems associated with cancer. In this context, advanced psychosocial care including proper counseling, education, assurance of continued support and different forms of psychotherapy should be offered to the cancer patients and their families.[6]

In addition to its uncontrolled local disease, cancer accompanies with a number of complications along with its treatment and clinical course. These complications include neurological, skeletal, hematological, gastrointestinal tract and respiratory complications. The management of these complications differs from each other, and may require admission to intensive care unit (ICU). In this regard, acute life-threatening illnesses associated with cancer require ICU admission. Most often, the patients with leukemia, lymphoma and lung cancer are encountered in ICU.[7] The 5\% and $15 \%$ of the patients with solid tumors and hematological malignancies require ICU admissions due to acute complications, respectively.[8] Similarly, $8 \%$ and $27 \%$ of the patients with lung cancer and other solid malignancies require ICU admissions, respectively.[8] Acute respiratory failure (ARF) occurs in 10-50\% patients with oncologic malignancies.[9] ARF is the most common cause of admission to ICU. In this paper, we will review only pulmonary complications requiring ICU admission in the patients with cancers.

\section{Search Strategy}

An online search was conducted on the most-trusted medical database "PubMed" and "Google Scholar" using keywords such as "Pulmonary Complications", "Cancer", "Intensive Care Unit", "ICU" or the combination of these words to collect articles related to our topic of interest. We read the articles thoroughly and carefully in order to write this review.

\section{Literature Review}

Pulmonary complications are common due to cancer and cancer treatment. For the way of explanation, pulmonary complications indicate a serious sequel of cancer or occur because of the therapies employed for the treatment of cancer. Pulmonary system is very sensitive to injuries caused by cancer therapy, resulting in fatal complications. These therapies may affect lung parenchyma, vasculature and pleural space.[9] Therefore, early detection of these complications and prompt treatment help improve prognosis and cancer-survival. Main reasons for the admission of cancer patients in ICU are acute respiratory failure (ARF), post-operative, infection and septic complications.[10,11]

\section{Acute Respiratory Failure (ARF)}

Acute respiratory failure (ARF) is a devastating condition in cancer patients where functions of lung or respiratory muscle pump are impaired, leading to high mortality.[12] About $15 \%$ of the patients with cancer suffer from ARF and require ICU admission where mortality rate of these patients is up to 50\%.[13] Mortality is even higher in those who require mechanical ventilation.[12] Frequent causes of ARF include pulmonary infections, pulmonary edema (cardiogenic or non-cardiogenic), lung injury due to cancer therapy, venous thromboembolism (VTE), diffuse alveolar hemorrhage (DAH) and direct involvement of lung tissues by cancer.[14]

Pulmonary Infections (Pneumonia): Pneumonia refers to lung infection that is caused by organisms such as bacteria, viruses or fungi. The patients with cancer are at risk of developing pneumonia due to immunosuppression and hospital mortality remains high due to multi-resistant pathogens.[15] Studies reveal that bacterial pneumonias complicate $10 \%$ of cancer patients admitted in hospital, especially of those with hematological malignancies, where in, it poses risk of developing pneumonia in $30 \%$ cases. Similarly, $80 \%$ of cancer patients who receive hematopoietic stem cell transplantation (HSCT) encounter an 


\section{International Journal of Innovative Research in Medical Science (IJIRMS) Volume 03 Issue 12 Dec 2018, ISSN: 2455-8737, Imp. Factor - 4.102 \\ Available online at $-\underline{w w w . i j i r m s . i n}$}

episode of pneumonia, causing death in 20\% of them.[16] Obstructive pattern of lung function is a risk factor of pneumonia in cancer patients undergoing cytotoxic chemotherapy, resulting in incidence of pneumonia in $19 \%$ of cases.[17] The most common organisms causing bacteria in non-neutropenic phase include Streptococcus pneumoniae, Haemophilis influenzae and respiratory viruses while P. aeruginosa, Enterobacteraceae, S. pneumonia, P. aeruginosa are commonly encountered in neutropneic phase of cancer.[18] Additionally, Penicillin-resistant pneumococcus is also predominant in cancer patients.

The cancer patients are susceptible to bacterial pneumonias due to immune dysregulation, neutropenia, aspiration events, architectural damage to lungs (mucositis, impaired airway protection, etc.) and malnutrition.[19] Bacterial pneumonia increases morbidity and mortality in cancer patients. Neutropenia and cytotoxic therapies may result in life-threatening pneumonias.[16] Pneumonia in cancer patients accounts for $50 \%$ of the patients with septic shock.[20] Severe pneumonia requires ICU admission due to its high morbidity and mortality.[21] A number of severity scores such as Pneumonia Severity Index and the CURB65 score have been designed to assess the severity of community-acquired pneumonia (CAP); however, these scores are either limited value or underused, respectively.[22] On the other hand, delayed ICU admission is associated with high mortality. Therefore, prompt recognition of the severity of CAP and urgent transfer to ICU improve the clinical outcome.[22] Although neutopenia is an important risk factor for developing serious infections; however, it has no effect on mortality in the cancer patients.[23] Invasive ventilation and septic shock at the time of ICU admission are associated with high mortality.[23] It has also been studied that the patients with cancer and CAP have worse outcome as compared to those without cancer.[24] The reasons why the patients with cancer and CAP have worse outcome include immune dysfunction, prior hospitalizations and previous use of antibiotics, making the patients prone to infections with difficult pathogens. Intensive care with non-invasive mechanical ventilation, prompt intubation along with broad-spectrum antibiotics and nursing care are of prime importance in the clinical outcome.

Pulmonary Edema: Pulmonary edema refers to the excess fluid in the lung and lung spaces. There are two types of pulmonary edema: cardiogenic and non-cardiogenic. Cardiogenic pulmonary edema develops due to elevated pulmonary capillary pressure caused by left-sided heart failure. Non-cardiogenic pulmonary edema develops due to increased capillary permeability caused by endothelial and epithelial damage.[25] Acute pulmonary edema is an emergency and one of the common complications developed in the patients with cancer.[26] It can be cardiogenic or noncardiogenic. Cardiogenic pulmonary edema in cancer patients may develop due to cardiomyopathy caused by certain drugs and radiotherapy used in cancer treatment. Similarly, non-cardiogenic pulmonary edema may develop due to acute reaction to the drugs (vinblatsine or mitomycin) or lung tissue damage due to cancer itself. Additionally, infections in cancer patients also cause noncardiogenic pulmonary edema. Acute respiratory distress syndrome and capillary leak syndrome are acute and serious conditions affecting the lungs in cancer patients.[26]

The patients with cancer and pulmonary edema should be admitted to ICU to intubate as some studies report positive outcome.[27] However, these studies have not specified the characteristics of the patients who should be intubated. In this context, Zarogoulidis et al.,[27] suggested that BMI $>25 \mathrm{~kg} / \mathrm{m}^{2}$ and low postoperative FEV1 can be used as predictive factors for the early admission to ICU. On the contrast, Griner.[28] recommends that appropriate treatment given to the patients with acute pulmonary edema in emergency room is sufficient, and only exceptionally ill patients should be admitted to ICU in order to make it cost-effective with short hospital stay. Other treatments of acute pulmonary edema include oxygen (if $\mathrm{SpO} 2<92 \%$ ), nitrates, diuretics, morphine and inotropes.[29]

Venous Thromboemolism (VTE): VTE is a serious problem in the patients with cancer or those who undergo surgical procedures. In spite of its high mortality, many oncologists underestimate the severity of VTE in cancer patients.[30] In fact, cancer is an independent and major risk factor of VTE. It poses 6-7-fold greater risk of thrombosis as compared to general population without cancer, leading to increased morbidity and mortality.[30,31] VTE in the patients with cancer occurs due to Virchow's triad: cancerinduced hypercoagulability, endothelial damage and stasis.[32] Deep vein thrombosis (DVT) and pulmonary embolism (PE) are two major forms of VTE. Their risk increases when the patients are admitted to ICU post-operatively.[31] Risk of recurrent cancerassociated VTE and chemotherapy-associated VTE can be predicted by using Ottawa score and Khoranna score, respectively.[32]

Treatment of VTE in the patients with cancer is critical due to high risk of recurrent VTE and serious hemorrhagic events. Additionally, co-morbid conditions are a challenge for the management of VTE. Currently available treatment for cancerassociated VTE recommends low molecular weight heparin (LMWH) for cancer-associated VTE for 3-6 months, unfractionated heparin (UFH) for renal insufficiency, warfarin and fondaparinux heparin-induced thrombocytopenia (HIT).[32,33] It has been observed that LMWH are more effective than warfarin with similar profile of side effects. However, warfarin can be employed as an alternative where LMWH is contraindicated. Regarding VTE prophylaxis, all the hospitalized patients with reduced mobility and no contraindication should receive VTE prophylaxis.[34]

Diffuse Alveolar Hemorrhage (DAH): DAH is a life-threatening pulmonary complication in a number conditions including cancer. Risk factors of DAH include infections, low platelets and coagulopathy.[35] The mechanism of DAH is suggested to be due to disruption of the alveolar-capillary basement membrane caused by injury or inflammation.[36] Clinical presentation of DAH is non-specific including pyrexia, cough, hemoptysis, dyspnea and chest pain. However, DAH is a well-recognized complication of acute myeloid leukemia (AML) and the clinicians should be aware of this condition while dealing with AML. The patients with DAH requiring ICU admission may be dealt with supplemental oxygen therapy, mechanical ventilation, reversal of coagulopathy, plasmapharesis, platelet transfusion and hemodynamic support.[35]

Direct Invasion of Cancer: Lungs are also affected by direct effect of cancer, especially lung cancer. Lung cancer can invade adjacent tissues such as vascular bundle and chest wall. Additionally, pressure effect disturbs functional-anatomic integrity and plays important role in respiratory compromise. 


\section{International Journal of Innovative Research in Medical Science (IJIRMS) Volume 03 Issue 12 Dec 2018, ISSN: 2455-8737, Imp. Factor - 4.102 \\ Available online at $-\underline{w w w . i j i r m s . i n}$}

Radiation Therapy Associated Pulmonary Complications: Radiation therapy is an important therapeutic modality used to treat cancer patients. It uses high dose of high-energy particles to damage cancer cells. Lung tissues (especially alveolar-capillary complex) are the most sensitive tissues to radiations. Along with damaging cancerous cells, radiation therapy damages the adjacent structures in the region of exposure. In fact, high-energy particles break strong chemical bonds releasing high reactive free radical species, which affect cellular components.[37] Radiation therapy can damage lungs in two phases: acute and late.[38] When a lung complication occurs before 6 months, it is called acute lung injury. Pneumonitis is an acute radiation-induced lung injury, which refers to inflammation of the lung tissue. Similarly, if a complication occurs after 6 months, it is called late complication. Fibrosis is a late radiation-induced lung injury that refers to scarring the lung tissues.

Treatment of radiation pneumonitis includes steroids, ACE inhibitors, pentoxyphylline and anti-oxidants.[38] Management of radiation fibrosis includes general supportive management (good nutrition, regular exercise, prophylactic vaccinations), clearing airway secretions (postural drainage, chest percussion, inhaled mannitol and hypertonic saline), anti-inflammatory agents (corticosteroids, antibiotics) and treatment of exacerbations.[38] Pneumonitis leads to respiratory failure warranting ICU admission where survival rate is improved.[39]

Chemotherapy-Related Pulmonary Complications: Chemotherapy may lead to direct or indirect potentially fatal complications.[40] It is a challenge to identify these complications early due to misinterpretation as other conditions like pulmonary edema, infections, and radiation pneumonitis. Chemotherapeutic agents may lead to cytotoxic pneumonitis, usual interstitial pneumonia (UIP) and drug-induced interstitial lung disease (DILD).[41] Primary management of chemotherapeutic pulmonary complications aims at suppressing inflammatory response and preventing continuing pulmonary fibrosis.[41]

\section{Conclusion}

Pulmonary complications in cancer patients are an additional challenge to healthcare system along with the management of primary malignancy. Most often, these pulmonary complications require critical care and admission to intensive care unit (ICU). Most common pulmonary complications among cancer patients are acute respiratory failure caused by various conditions such as pneumonia, pulmonary edema, VTE, DAH and direct invasion of lung tissues, and complications arising due to radiation therapy and chemotherapy. However, management of these complications in ICU is still controversial due to longer hospital stay and higher cost. Therefore, authors suggest ICU admissions only for those who are exceptionally ill. Moreover, there is need to evaluate outcome of ICU admissions for pulmonary complications in cancer patients in terms of short-term survival and overall mortality while considering the longer hospital stay and healthcare costs.

\section{References}

[1] Fouad YA, Aanei C. Revisiting the hallmarks of cancer. Am J Cancer Res 2017;7(5):1016-36.

[2] Nagata S, Tanaka M. Programmed cell death and the immune system. Nat Rev Immunol 2017;17(5):333-40.
[3] Labi V, Erlacher M. How cell death shapes cancer. Cell Death Dis. 2015;6(3):e1675.

[4] Cancer [Internet]. Sep 12, 2018 [cited Sep 24, 2018]. Available from: http://www.who.int/news-room/factsheets/detail/cancer.

[5] Naughton MJ, Weaver KE. Physical and mental health among cancer survivors: considerations for long-term care and quality of life. N C Med J. 2014;75(4):283-6.

[6] Grassi L, Spiegel D, Riba M. Advancing psychosocial care in cancer patients. F1000Res 2017;6:2083.

[7] Freedman N. Prognosis of cancer patients in the intensive care unit [Internet]. Mar 23, 2018 [cited Sep 24, 2018]. Available from: https://www.uptodate.com/contents/prognosis-of-cancerpatients-in-the-intensive-care-unit.

[8] Soares M, Toffart AC, Timsit JF, Burghi G, Irrazabal C, Pattison $\mathrm{N}$, et al. Intensive care in patients with lung cancer: a multinational study. Ann Oncol 2014;25(9):1829-35.

[9] Abouzgheib W, Dellinger RP. Pulmonary Complications in Cancer Patients. In: Todd K, Thomas Jr C. (eds). Oncologic Emergency Medicine. Springer, Cham; 2016.

[10] Schellongowski P, Sperr WR, Wohlfarth P, Knoebl P, Rabitsch W, Watzke H, et al. Critically ill patients with cancer: chances and limitations of intensive care medicine-a narrative review. ESMO Open 2016;1(5):e000018.

[11] Kostakou E, Rovina N, Kyriakopoulou M, Koulouris NG, Koutsoukou A. Critically ill cancer patient in intensive care unit: issues that arise. J Crit Care 2014;29(5):817-22.

[12] Scala R, Heunks L. Highlights in acute respiratory failure. Eur Respir Rev 2018;27(147):pii:180008.

[13] Azoulay E, Schlemmer B. Diagnostic strategy in cancer patients with acute respiratory failure. Intensive Care Med 2006;32(6):808-22.

[14] Pastores SM, Voigt LP. Acute respiratory failure in the patient with cancer: diagnostic and management strategies. Crit Care Clin 2010;26(1):21-40.

[15] Rabello LS, Lisboa T, Soares M, Salluh JI. Personalized treatment of severe pneumonia in cancer patients. Expert Rev Anti Infect Ther 2015;13(11):1319-24.

[16] Wong JL, Evans SE. Bacterial pneumonia in cancer patients: novel risk factors and current management. Clin Chest Med 2017;38(2):263-77.

[17] Watanabe K, Shinkai M, Tei Y, Kaneko T. Chemotherapy in Non-Small Cell Lung Cancer Patients Receiving Oxygen Therapy. Oncol Res Treat 2016;39:587-90.

[18] Kufe DW, Pollock RE, Weichselbaum RR, Holland-Frei Cancer Medicine. 6th edition. Hemilton: BC Decker, 2003.

[19] Wong A, Marrie TJ, Garg S, Kellner JD, Tyrrell GJ, SPAT Group.. Increased risk of invasive pneumococcal disease in haematological and solid-organ malignancies. Epidemiol Infect 2010;138(12):1804-10.

[20] Rabello LSCF, Azevedo LCP, de Souza IAO, Torres VBL, Rosolem MM, Lapa e Silva1 JR, et al. Severe pneumonia in critically ill cancer patients: clinical outcomes and a comparison between healthcare- 


\section{International Journal of Innovative Research in Medical Science (IJIRMS) Volume 03 Issue 12 Dec 2018, ISSN: 2455-8737, Imp. Factor - 4.102 Available online at $-\underline{w w w . i j i r m s . i n}$}

associated pneumonia and community-acquired pneumonia. Crit Care 2013;17(Suppl 4):P28.

[21] Lim HF, Phua J, Mukhopadhyay A, Ngerng WJ, Chew MY, Sim TB, et al. IDSA/ATS minor criteria aid preintensive care unit resuscitation in severe communityacquired pneumonia. Eur Res J 2014;43:852-62.

[22] Chalmers JD. ICU admission and severity assessment in community-acquired pneumonia. Crit Care 2009;13(3):156.

[23] Leoni D, Encina B, Rello J. Managing the oncologic patient with suspected pneumonia in the intensive care unit. Exp Rev Anti-inf Ther 2016;14(10):943-60.

[24] Jose RJP, Mohammed AO, Goldring JJP, Chambers RC, Brown JS, Agarwal B. Cancer patients with communityacquired pneumonia treated in intensive care have poorer outcomes associated with increased illness severity and septic shock at admission to intensive care: a retrospective cohort study. Pneumonia 2015;6:6010077.

[25] Murray JF. Pulmonary edema: pathophysiology and diagnosis. Int J Tuberc Lung Dis 2011;15(2):155-60.

[26] Stover DE, Kaner RJ. Pulmonary complications in cancer patients. CA: Cancer J Clin 1996;46(5):303-20.

[27] Zarogoulidis P, Pataka A, Terzi E, Hohenforst-Schmidt $\mathrm{W}$, Machairiotis N, Huang $\mathrm{H}$, et al. Intensive care unit and lung cancer: when should we intubate? J Thorac Dis. 2013;5(Suppl 4):S407-12.

[28] Griner RF. Treatment of acute pulmonary edema: conventional or intensive care? Ann Int Med 1972;77(4):501-6.

[29] Purvey M, Allen G. Managing acute pulmonary oedema. Aust Prescr 2017; 40(2):59-63.

[30] Khalil J, Bensaid B, Elkacemi H, Afif M, Bensaid Y, Kebdani $\mathrm{T}$, et al. Venous thromboembolism in cancer patients: an underestimated major health problem. World J Surg Oncol 2015;13:204.

[31] Xu JX, Dong J, Ren H, Chen XJ, Yang Y, Chen RX, et al. Incidence and risk assessment of venous thromboembolism in cancer patients admitted to intensive care unit for postoperative care. J BUON 2018;23(1):500-6.
[32] Ha N, Barnes GD. Optimal Treatment Approaches of Cancer-Induced Thrombosis [Internet]. Jun 15, 2017 [cited Sep 29, 2018]. Available from: https://www.acc.org/latest-incardiology/articles/2017/06/14/13/58/optimal-treatmentapproaches-of-cancer-induced-thrombosis.

[33] Lee AY, Peterson EA. Treatment of cancer-associated thrombosis. Blood 2013;122:2310-7.

[34] Bell J, O'Rourke C. Prophylaxis and treatment of venous thromboembolism in cancer patients [Internet]. Jan 2016 [cited Sep 29, 2018]. Available from: https://www.thehospitalist.org/hospitalist/article/121939/prophylaxisand-treatment-venous-thromboembolism-cancer-patients.

[35] Nanjappa S, Jeong DK, Muddaraju M, Jeong K, Hill ED, Greene JN. Diffuse alveolar hemorrhage in acute myeloid leukemia. Cancer Control 2016;23(3):272-7.

[36] Schwarz MI. The diffuse alveolar hemorrhage syndromes [Internet]. Aug 2018 [cited Sep 29, 2018]. Available from: https://www.uptodate.com/contents/thediffuse-alveolar-hemorrhage-syndromes.

[37] Olivier KR, Jett JR, Hollingsworth H. Radiation-induced lung injury [Internet]. Aug 2018 [cited Sep 29, 2018]. Available at: https://www.uptodate.com/contents/radiation-inducedlung-injury.

[38] Giridhar P, Mallick S, Rath GK, Julka PK. Radiation induced lung injury: prediction, assessment and management. Asian Pac J Cancer Prev 2015;16(7):26137.

[39] Andréjak C, Terzi N, Thielen S, Bergot E, Zalcman G, Charbonneau P, et al. Admission of advanced lung cancer patients to intensive care unit: a retrospective study of 76 patients. BMC Cancer 2011;11:159.

[40] Becker A, Frauenfelder T. Pulmonary complications in chemotherapy. Radiologe 2014;54(10):1023-38.

[41] Schwaiblmair M, Behr W, Haeckel T, Märkl B, Foerg W, Berghaus T. Drug induced interstitial lung disease. Open Respir Med J 2012;6:63-74. 